\title{
A Lagrangian parcel based mixing plane method for calculating water based mixed phase particle flows in turbo-machinery
}

\author{
Colin S. Bidwell
}

Received: 19 August 2014 / Revised: 15 January 2015 / Accepted: 23 January 2015 / Published online: 15 February 2015

(C) OWZ (outside the USA) 2015

\begin{abstract}
A method for calculating particle transport through turbo-machinery using the mixing plane analogy was developed and used to analyze the energy efficient engine $\left(E^{3}\right)$. This method allows the prediction of temperature and phase change of water based particles along their path and the impingement efficiency and particle impact property data on various components in the engine. This methodology was incorporated into the LEWICE3D V3.5 software. The method was used to predict particle transport in the low pressure compressor of the $\mathrm{E}^{3}$. The $\mathrm{E}^{3}$ was developed by NASA and GE in the early 1980s as a technology demonstrator and is representative of a modern high bypass turbofan engine. The $\mathrm{E}^{3}$ flow field was calculated using the NASA Glenn ADPAC turbo-machinery flow solver. Computations were performed for a Mach 0.8 cruise condition at $11,887 \mathrm{~m}$ assuming a standard warm day for ice particle sizes of 5, 20 and 100 microns and a free stream particle concentration of $0.3 \mathrm{~g} / \mathrm{m}^{3}$. The impingement efficiency results showed that as particle size increased average impingement efficiencies and scoop factors increased for the various components. The particle analysis also showed that the amount of mass entering the inner core decreased with increased particle size because the larger particles were less able to negotiate the turn into the inner core due to particle inertia. The particle phase change analysis results showed that the larger particles warmed less as they were transported through the low pressure compressor. Only the smallest 5 micron particles were warmed enough to produce melting with a maximum average melting fraction of 0.18 . The results also showed an appreciable amount of particle sublimation and evaporation for the 5 micron particles entering the engine core $(22.6 \%)$.
\end{abstract}

C. S. Bidwell ( $\square)$

Icing Branch, NASA Glenn Research Center,

21000 Brookpark Rd, Cleveland, OH 44135, USA

e-mail: colin.s.bidwell@nasa.gov

\section{Introduction}

Over the last several years work has been ongoing to develop tools to analyze aircraft configurations subjected to supercooled large droplet (SLD) conditions [1] and high ice water content (HIWC) conditions [2]. Both the SLD and HIWC environments contain conditions outside of the FAA Appendix C [3] Certification Envelope. The SLD environment contains larger drops than the Appendix $\mathrm{C}$ envelope which impinge further back and which are subject to breakup and splash at impact. The HIWC environment contains large ice or mixed phase particles ( $>50$ micron) in very high concentrations $\left(\sim 10 \mathrm{~g} / \mathrm{m}^{3}\right)$ up to very high altitudes $(\sim 40,000$ feet). The HIWC environment has been responsible for many engine anomalies including engine rollbacks and shutdowns [2]. Work is underway to quantify the HIWC environment and to develop ground test facilities and computational tools to assess the sensitivity of various engines to the HIWC threat. New certification rules, which will require aircraft to fly safely through these conditions, are on the horizon. These new rules will generate new requirements for aircraft ice protection system design and certification and for the tools which aid in these processes.

The development of icing computational analysis tools which produce sufficiently accurate results in a reasonable amount of computational time for turbo-machinery flows has been a major challenge $[4,5]$. The use of unsteady analysis tools to simulate the flow and particle transport in highly time dependent turbo-machinery flows is prohibitively expensive and possibly unnecessary. For this reason a methodology was developed at NASA Glenn which uses the steady flow, mixing plane assumption commonly used in turbo-machinery design tools.

Mixing plane methods have been used to analyze and design turbo-machinery for many years. The methods use a 
steady state flow assumption to calculate flow in the passages between blades. The flow variables at the passage exit are circumferentially averaged to generate circumferentially uniform values for use as boundary conditions to the downstream blade row. This technique is used in a marching scheme starting at the most upstream component and traversing through the engine to the most downstream component to generate the flow through the engine. In some schemes multiple passes through the engine are made through the engine to improve accuracy $[6,7]$.

The mixing plane analogy has been used to develop the particle transport method described herein. The method calculates parcel paths, which are used to represent the paths of collection of particles with the same properties, through the blade passages using a Lagrangian based Adams-type, variable time step, predictor-corrector integration scheme. The parcel velocity, temperature, melt ratio and concentration data are circumferentially averaged at the blade passage exit to generate initial conditions for the parcels in the downstream row. The method is employed in a marching fashion whereby particle transport is calculated first in the most upstream zone and then in successive zones to the engine exit.

\section{Numerical method}

\subsection{Lagrangian parcel based trajectory method}

The trajectory algorithm used in the LEWICE3D program is a Lagrangian based Adams-type predictor-corrector integration method with variable time step. The original formulation of the particle trajectory equations was by Norment [8]. Bidwell added energy and mass transfer equations to model phase change of water based particles [9]. The equations are integrated using the DVDQ scheme developed by Krogh [10]. The integration scheme has the ability to test for computational stability and machine precision to estimate round-off error.

The formulation of the particle trajectory equations by Norment and Bidwell assumes the particle concentrations are small and that there is no momentum transfer between the gas and particle phases. The buoyancy forces are neglected for the air-water particle mixture and the particles are assumed to be spherical or near spherical such that the only aerodynamic force acting on the particles is drag. The formulation chosen by Norment for the momentum equations incorporates the Froude number, Davies Number, Reynolds number and the still air terminal settling speed of the particle in air to maximize generality and minimize computational effort. The nondimensional momentum equation developed by Norment for a non-moving reference frame was;

$\frac{d \overrightarrow{\mathrm{V}}_{p}}{d \tau}=\frac{1}{N_{f}}\left[\left[\frac{N_{D} N_{R, S}}{V_{S} N_{D, S} N_{R}}\left(\vec{V}_{a}-\vec{V}_{p}\right)-\vec{K}\right]\right.$ where we have the defined quantities,

$N_{D}=C_{D} N_{R}^{2} \quad$ Davies number

$N_{f}=\frac{V_{\infty}^{2}}{L g} \quad$ Froudenumber

$N_{R}=\frac{\rho_{a} d V_{\infty}}{\mu_{a}}\left|\vec{V}_{a}-\vec{V}_{p}\right|$ particle Reynolds number

The air velocity, $\vec{V}_{a}$, and particle velocity, $\vec{V}_{p}$, are nondimensonalized by the free stream speed, $V_{\infty}$, and time, $\tau$, has been non-dimensionalized by the reference length divided by the free stream speed $\left(L / V_{\infty}\right)$. The values of $N_{R, s}$ and $N_{D, s}$ represent the particle Reynolds number and Davies number based on the still air settling speed of the particle, $V_{s}$, respectively. The Froude number is the ratio of inertial to gravitational forces. The Froude number is a function of the gravitational constant, $g$, the characteristic dimension of the body, $L$, and the free stream speed, $V_{\infty}$. The particle Reynolds number is calculated using the particle diameter, $\mathrm{d}$, and the local air density, $\rho_{\mathrm{a}}$, and viscosity, $\mu_{\mathrm{a}}$. The gravitational force operates in the unit direction, $\vec{K}$. For a rotating reference frame with a constant rotational speed, $\Omega$, about the X-axis, which has been non-dimensionalized by $L / V_{\infty}$, the modified trajectory equations are:

$$
\begin{aligned}
\frac{d \overrightarrow{\mathrm{V}}_{p}}{d \tau}= & \frac{1}{N_{f}}\left[\left[\frac{N_{D} N_{R, S}}{V_{S} N_{D, S} N_{R}}\left(\vec{V}_{a}-\vec{V}_{p}\right)-\vec{K}\right]\right. \\
& +2\left(\vec{\omega}_{p} \times \vec{V}_{p}\right)-\Omega^{2} \vec{r}
\end{aligned}
$$

In the above equation $\vec{r}$ is the position vector in the radial direction normal to the $\mathrm{x}$-axis non-dimensionalized by $L$. The angular velocity of the particle, $\vec{\omega}_{p}$, has been nondimensionalized by $L / V_{\infty}$.

The mass and energy equations developed for the Glenn Phase Change Model [5] were incorporated into the LEWICE3D V3.5 software. The formulation assumes:

(1) low particle concentration, such that there is no transfer of mass or energy between the gas and particle phases

(2) the mass and energy transfer are in equilibrium and hence the particle surface pressure is at the saturation pressure for the given surface temperature

(3) the thermal and kinetic energy do not convert from one to the other

(4) the surface of the particle is considered to be liquid until it is fully solidified

(5) the gasses are ideal, the thermodynamic properties of the dry and humid gas are equivalent

(6) the gas is extremely clean hence only condensation is possible on drops and the gas can become supersaturated

(7) the particles are assumed to be spherical with a homogenous temperature 
The mass balance for the Glenn Model is:

$\frac{d m_{p}}{d \tau}=\pi d^{2} h_{m} \rho_{a}\left(\omega_{\infty}-\omega_{p, s a t}\right)$

Where we have the defined quantities,

$$
\begin{aligned}
\omega & =\frac{M_{w}}{M_{a}\left(\frac{\phi P_{a m b}}{P_{\text {sat }}}-1\right)} \\
h_{m} & =\frac{I_{p, a}}{d}\left[2+0.6 N_{R}^{1.5} \sqrt{S_{c}}\right] \\
S_{c} & =\frac{\mu_{a}}{\rho_{a} I_{p, a}} \quad \text { Schmidt number }
\end{aligned}
$$

The particle mass rate of change, $\frac{d m_{p}}{d \tau}$, is calculated from the evaporation potential, $\omega_{\infty}-\omega_{p, s a t}$, and mass transfer coefficient, $h_{m}$, which is determined by analogy to the momentum equation using the Schmidt number. The evaporation potential is calculated as the difference between the free stream specific humidity, $\omega_{\infty}$, and the local specific humidity of air at saturation, $\omega_{p, \text { sat }}$. Equation 7 defines the relation between the specific humidity, the local ambient pressure, $P_{a m b}$, saturation pressure, $P_{s a t}$, and relative humidity, $\phi$, and the molar mass of air and water $\left(M_{a}, M_{w}\right)$. The Schmidt number, which is the ratio of the momentum and mass diffusivity, is dependent upon the air viscosity and density and the mass diffusivity of water in air, $I_{p, a}$.

The energy equation for the Glenn Model is:

$$
\begin{gathered}
\frac{d T_{p}}{d \tau}=0, \quad T_{p}=273.15 \mathrm{~K}, \\
\text { particle is a mixture of solid and liquid } \\
\frac{d T_{p}}{d \tau}=\frac{6 L}{\pi \rho_{p} d^{3} V_{\infty} C_{p, p}}\left[\pi d^{2} h_{q}\left(T_{a}-T_{p}\right)+L_{v} \frac{d m_{p}}{d \tau}\right], \\
\begin{array}{c}
\text { particle is liquid } \\
\frac{d T_{p}}{d \tau}=
\end{array} \begin{array}{c}
\frac{6 L}{\pi \rho_{p} d^{3} V_{\infty} C_{p, p}}\left[\pi d^{2} h_{q}\left(T_{a}-T_{p}\right)+L_{u} \frac{d m_{p}}{d \tau}\right], \\
\text { particle is solid }
\end{array}
\end{gathered}
$$

Where we have the defined quantities,

$$
\begin{aligned}
& h_{q}=\frac{k_{a}}{d}\left[2+0.6 N_{R}^{1.5} \sqrt{P_{r}}\right] \\
& P_{r}=\frac{C_{p, a} \mu_{a}}{k_{a}} \quad \text { Prandt number }
\end{aligned}
$$

The time rate of change of the temperature, $\frac{d T_{p}}{d \tau}$, for the water based particles is different depending upon whether the particle is solid, liquid or a mixture of solid and liquid. When the particle is a mixture of water and ice it assumed to be at the freezing temperature of water. The temperature equations for the solid and liquid states are similar except that the equation for liquid has an evaporation term $\left(L_{v} \frac{d m_{p}}{d \tau}\right)$ while that for the solid has a sublimation term $\left(L_{u} \frac{d m_{p}}{d \tau}\right)$. The evaporation term involves the latent heat of vaporization of water, $L_{v}$, while the sublimation term involves the latent heat of sublimation of ice, $L_{u}$. The time rate of change of temperature for the particle in the solid and liquid states is dependent upon the local air and particle temperature difference $\left(T_{a}-T_{p}\right)$, the density, $\rho_{\mathrm{p}}$, and specific heat, $C_{p, p}$, of the particle, and the convective heat transfer coefficient, $h_{q}$, which is determined by analogy to the momentum equation through the Prandtl number. The Prandtl number, which is defined as the ratio of momentum diffusivity to thermal diffusivity, is dependent upon the specific heat, $C_{p, a}$, thermal conductivity, $k_{a}$, and viscosity of dry air.

The particle equations can be initialized in several ways depending upon the software settings and the origin of the particle. Initial conditions are required for particle size, velocity, temperature, and melt fraction. The initial conditions are either set by the user, set to free stream equilibriums values, set to inflow mixing plane values or generated by a rebound or splash model. The default setting for external aircraft calculations initializes the particle in the far-field with the still air, terminal particle settling speed.

\subsection{Rebound model}

A simple rebound model was incorporated into the LEWICE3D V3.5 software to analyze particle bounce from solid surfaces. The model assumes spherical particles with no rotation and no breakup or deformation. The rebound model relates the particle velocity before impact, $\vec{V}_{i}$, to that after impact, $\vec{V}_{r}$, using a model based on the coefficient of restitution, $C_{R}$, and the coefficient of dynamic friction, $C_{D F}$. The normal and tangential components of velocity of the rebounding particle from the model are:

$$
\begin{aligned}
\vec{V}_{r, n} & =-C_{R} \vec{V}_{i, n} \\
\vec{V}_{r, t} & =\vec{V}_{i, t}-C_{D F}\left(1+C_{R}\right) \vec{V}_{r, n}
\end{aligned}
$$

Where the subscripts, $n$, and, $t$, denote the normal and tangential directions respectively. The velocities in the above equations are non-dimensionalized by $V_{\infty}$.

\subsection{SLD splashing model}

The LEWICE3D V3.5 software incorporates the SLD splashing model developed by Wright [11]. For sufficiently large droplet size and impact velocity liquid particles can break apart on impact. For these cases the surface tension forces are not sufficient to overcome the impact forces. The resulting impact can result in some liquid remaining on the surface and a portion rebounding from the surface. The empirical LEWICE SLD splashing model (Eqs. 17-25) correlates the rebounding droplet size, velocity, rebound angle and mass fraction to the incoming particle properties at impact. The LEWICE splashing threshold parameter, $\mathrm{K}_{\mathrm{L}, \mathrm{n}}$, is used to define the breakup limit (Eq. 17). 


$$
\begin{aligned}
& \mathrm{K}_{L, n}=\left(\sin \theta_{i}\right)^{-1.25} \sqrt{K^{*}} f^{*-3 / 8}>200 \\
& \text { splashing threshold parameter } \\
& \frac{d_{r}}{d_{i}}= 8.72 e^{-.0281 K^{*}}, \quad 0.05 \leq \frac{d_{r}}{d_{i}} \leq 1 \\
& \frac{m_{r}}{m_{i}}= 0.9\left[1.0-\sin \theta_{i}\right]\left[1.0-e^{\left(-.0092\left(K_{L, n}-200\right)\right)}\right] \\
& \vec{V}_{r, n}=-\left(0.3-0.002 \theta_{i}\right) \vec{V}_{i, n} \\
& \vec{V}_{r, t}=\left(1.075-0.0025 \theta_{i}\right) \vec{V}_{i, t}
\end{aligned}
$$

where we have the defined quantities,

$$
\begin{aligned}
f^{*}= & \frac{3}{2}\left(\frac{L W C}{\rho_{w}}\right)^{1 / 3} \text { dimensionless drop frequency } \\
K= & \left(\frac{\rho_{w}^{3} d_{i}^{3}\left|\vec{V}_{i, n}\right|^{5}}{\sigma^{2} \mu_{w}}\right)^{1 / 4} \\
& \text { Mundo splashing parameter } \\
K^{*}= & \left(\frac{\rho_{w}^{3} d_{i}^{3}\left|\vec{V}_{i, n} \sin \theta_{i}\right|^{5}}{\sigma^{2} \mu_{w}}\right)^{1 / 4}=K\left(\sin \theta_{i}\right)^{1.25} \\
\theta_{i}= & \begin{array}{l}
\text { angle between particle path tangent and surface } \\
\text { normal at impact }
\end{array}
\end{aligned}
$$

The splashing model assumes a group of particles with an average diameter, $d_{r}$, results from the impact and breakup of the incoming particle group of size, $d_{i}$, based on an empirical relation with the modified Mundo splashing parameter, $K^{*}$. The empirical splashing model also relates the splashed to incoming mass ratio $\left(m_{r} / m_{i}\right)$ to the splashing impact parameter, $K_{L, n}$, and the particle impact angle, $\theta_{i}$. The particle impact angle is also used to relate the particle veloc- ity before impact, $\vec{V}_{i}$, to that after impact, $\vec{V}_{r}$. The velocities in the above equations are non-dimensionalized by $V_{\infty}$. The splashing threshold parameter, which defines the boundary between splashing impacts and non-splashing impacts, is dependent upon the non-dimensional drop frequency, $f^{*}$, the droplet impact angle, and the modified Mundo splashing parameter. The non-dimensional drop frequency, which is a measure of the impinging mass on the surface, is a function of the liquid water content (LWC) of the air water mixture and the density of water, $\rho_{\mathrm{w}}$. Mundo used the splashing parameter, $K$, to define the splashing limit in his experiments which were performed at smaller impact velocities than for those observed in aircraft icing. The Mundo splashing parameter is dependent upon the droplet diameter, density, viscosity, $\mu_{\mathrm{w}}$, surface tension, $\sigma$, and the normal impact velocity, $\vec{V}_{i, n}$. The modified Mundo parameter uses a modified form of the impact velocity in the Mundo splashing parameter which was found by Wright to generate better comparisons to experimental impingement data for aircraft icing which typically occurs at higher speeds than those of the Mundo experiments.

\subsection{Mixing plane treatment}

The LEWICE3D V3.5 software uses a parcel based trajectory method for the calculation of collection efficiency and particle properties in turbo-machinery. Trajectories of parcels, which represent a collection of particles with the same properties, are calculated through each passage in the flow domain (Fig. 1). The collection efficiency for a segment at the exit of the blade passage or mixing plane is calculated based on the total mass of the parcels impacting a segment at a radial location and the area of the segment (Eq. 28; Figs. 2, 3). The parcel properties at the mixing plane are calculated using a mass weighted average of the properties of the particles

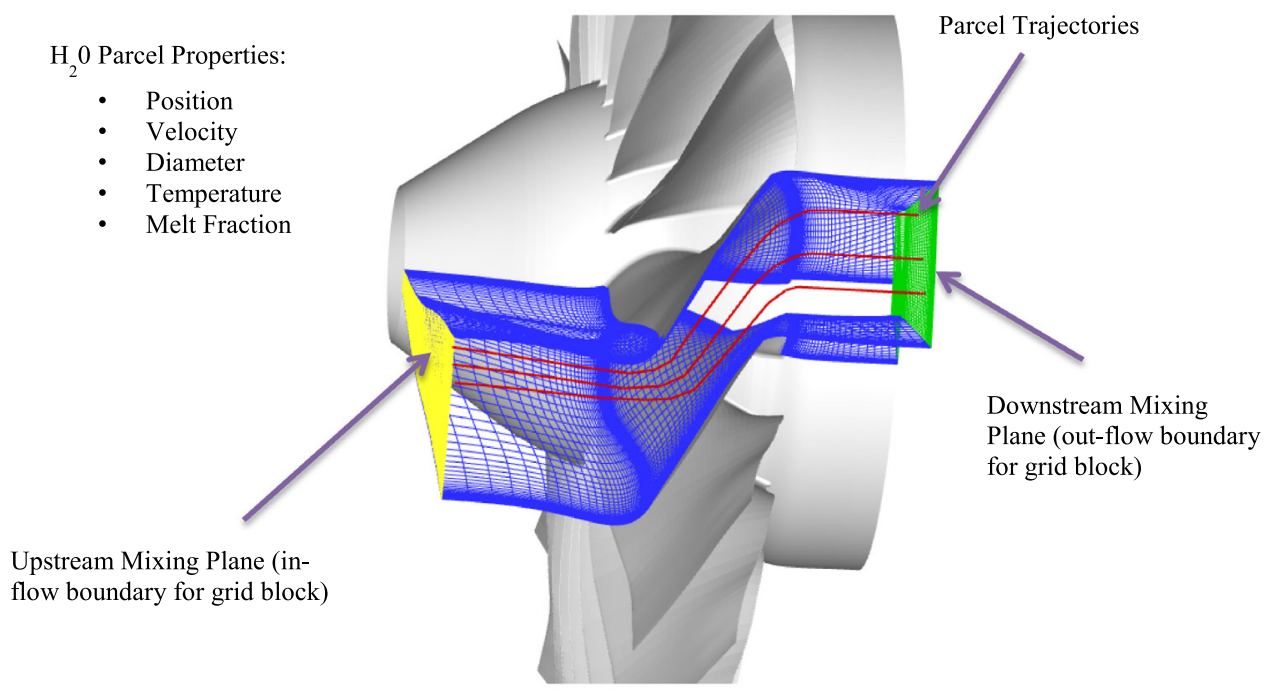

Fig. 1 Schematic of parcel trajectories in flow domain between two mixing planes 


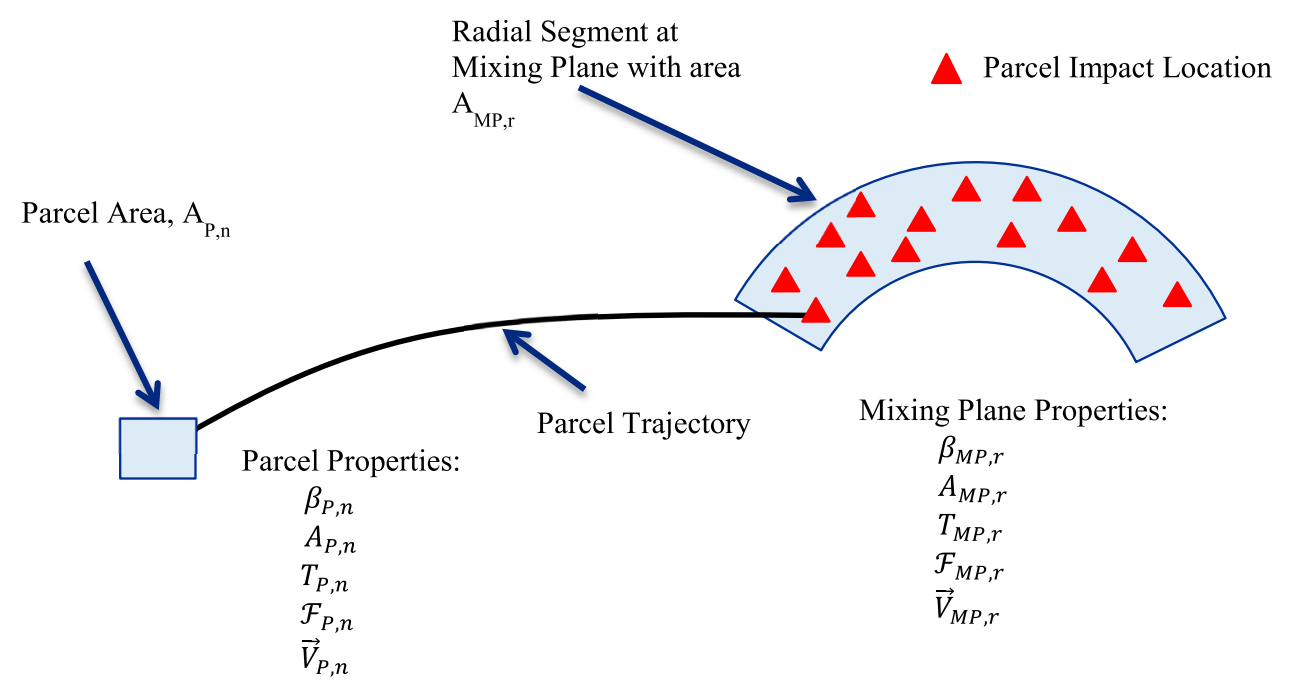

Fig. 2 Parcel contributions to particle state at downstream mixing plane

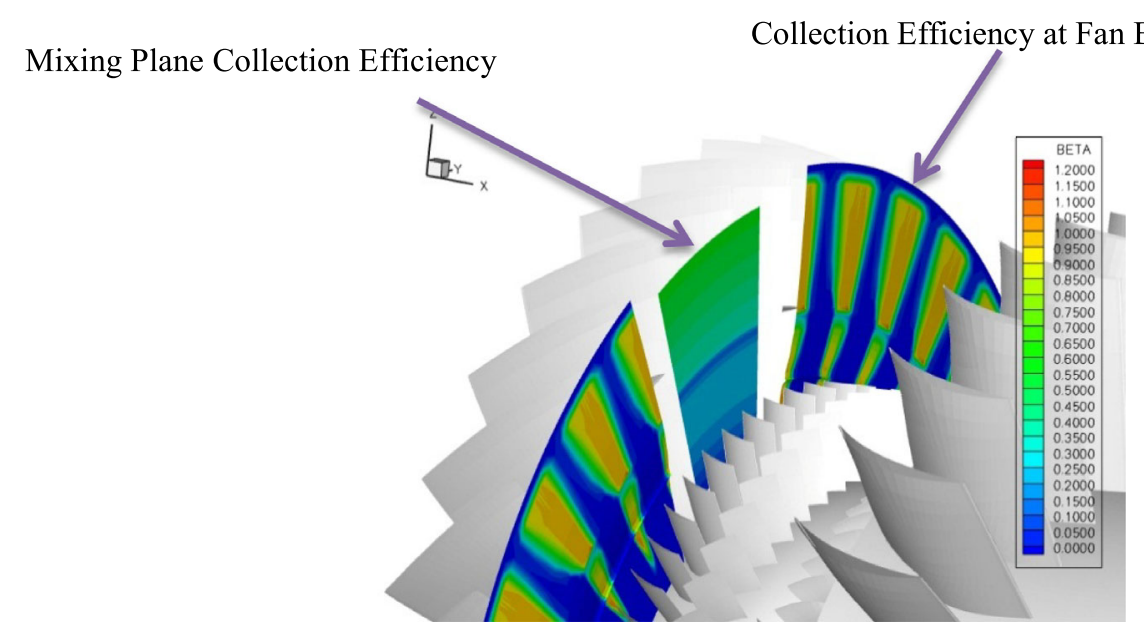

Fig. 3 Illustration of circumferential averaging of collection efficiency at fan exit mixing plane

parcels impacting a segment at a radial location (Eq. 26-29). The radial collection efficiency and particle property distributions generated at the mixing plane are used to generate initial conditions for the parcel mass and properties in the downstream passage associated with the mixing plane.

$$
\begin{aligned}
\beta_{M P, r} & =\frac{\sum_{n=1}^{N_{M P, r}} A_{P, n} \beta_{P, n}}{A_{M P, r}} \\
\vec{V}_{M P, r} & =\frac{\sum_{n=1}^{N_{M P, r}} A_{P, n} \vec{V}_{P, n}}{A_{M P, r}} \\
T_{M P, r} & =\frac{\sum_{n=1}^{N_{M P, r}} A_{P, n} T_{P, n}}{A_{M P, r}} \\
\mathcal{F}_{M P, r} & =\frac{\sum_{n=1}^{N_{M P, r}} A_{P, n} \mathcal{F}_{P, n}}{A_{M P, r}}
\end{aligned}
$$

The above equations relate the properties of collection efficiency, $\beta_{M P, r}$, velocity, $\vec{V}_{M P, r}$, temperature, $T_{M P, r}$, and melt fraction, $\mathcal{F}_{M P, r}$, at a radial location, $\mathrm{r}$, in the mixing plane to the a mass weighted average of the parcel properties of collection efficiency, $\beta_{P, n}$, velocity, $\vec{V}_{P, n}$, temperature, $T_{P, n}$, and melt fraction, $\mathcal{F}_{P, n}$, of the parcels that impact in the mixing plane segment at a radial location, $r$. In the above equation, $N_{M P, r}$ is the number of parcels that impact the mixing plane segment at a radial location, $\mathrm{r}$, and, $A_{M P, r}$, and, $A_{P, n}$, are the areas of the mixing plane segment at a radial location, $\mathrm{r}$, and the area of a parcel impacting in the mixing plane segment respectively. The velocities in the above equations have been non-dimensionalized by $V_{\infty}$.

\section{Results}

The mixing plane methodology was incorporated into the LEWICE3D V3.5 software [12]. The method was used to analyze the low pressure compressor of the $\mathrm{E}^{3}$ engine [13, 14]. The GRIDGEN [15] package was used to develop the grids for the engine and the ADPAC flow solver [16] was used to generate the flow solution for the analysis. 


\subsection{Particle calculations}

The LEWICE3D V3.5 grid based code incorporates droplet trajectory, heat transfer and ice shape calculation into a single computer program [12]. Version 3.5 of the LEWICE3D software, which incorporates several new features, was used for the analysis. These features include a new particle splash and bounce algorithm, a new geometry handling scheme which allows complex mirroring, transformation and relative motion of input grid blocks and a new algorithm which calculates zone to zone collection efficiencies using a mixing plane approach. These new additions enable users to analyze SLD and HIWC conditions and to calculate collection efficiency with particle splash and bounce effects through turbo-machinery.

\subsection{Grid and flow calculations}

GRIDGEN was used to develop the three-dimensional grids for the geometry [15]. The ADPAC flow solver [16] was used to generate the flow solution for the analysis. The ADPAC code is a three-dimensional, finite volume based, Reynolds Averaged Navier-Stokes flow solver. The code computes flows on complex propulsion system configurations using multi-block body fitted grids. The method employs a "mixing-plane" procedure to pass boundary condition data between grid blocks for the steady state flows. The code supports parallel computing and uses a Baldwin-Lomax based turbulence model.

\subsection{Configuration}

The $\mathrm{E}^{3}$ engine $[13,14]$ was selected as a test case for the newly developed mixing plane particle transport method incorporated into LEWICE3D V3.5. The $\mathrm{E}^{3}$ was developed by NASA and GE in the early 1980s as a technology demonstrator. The engine was chosen because it is representative of a modern high bypass turbofan engine, the geometry and experimental data were publicly available, and flow solutions were readily available.

\subsection{Analysis}

The $\mathrm{E}^{3}$ analysis included the calculation of flow and ice particle transport properties. The results for the flow are presented along with particle analysis for 5, 20, and 100 microns ice particle sizes.

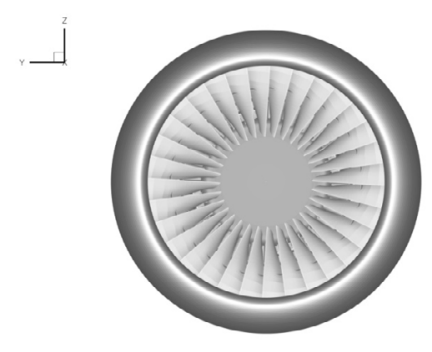

(a)

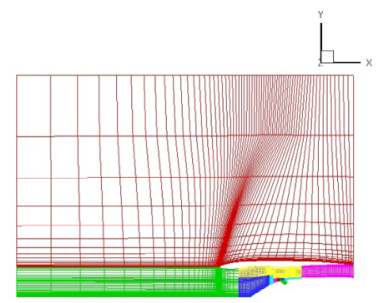

(b)

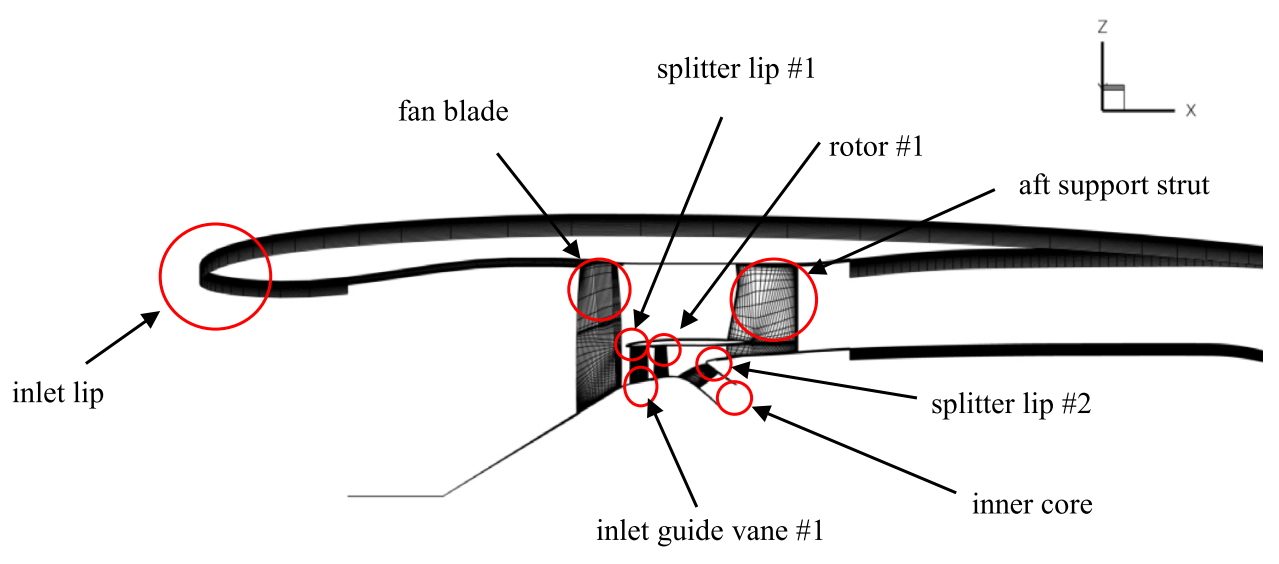

(c)

Fig. 4 Surface model and grid structure for $E^{3}$ flow model. a Surface model. b Grid block structure. c Element designation 


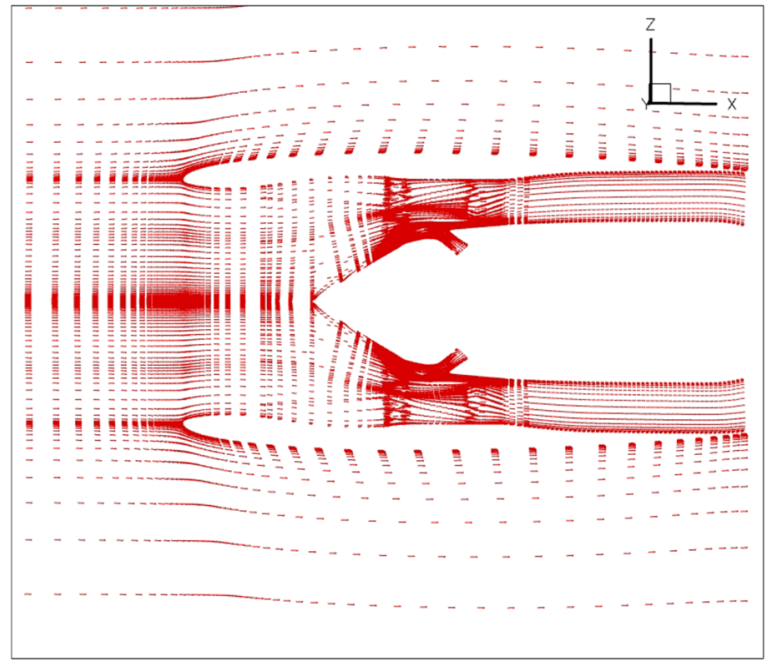

(a)

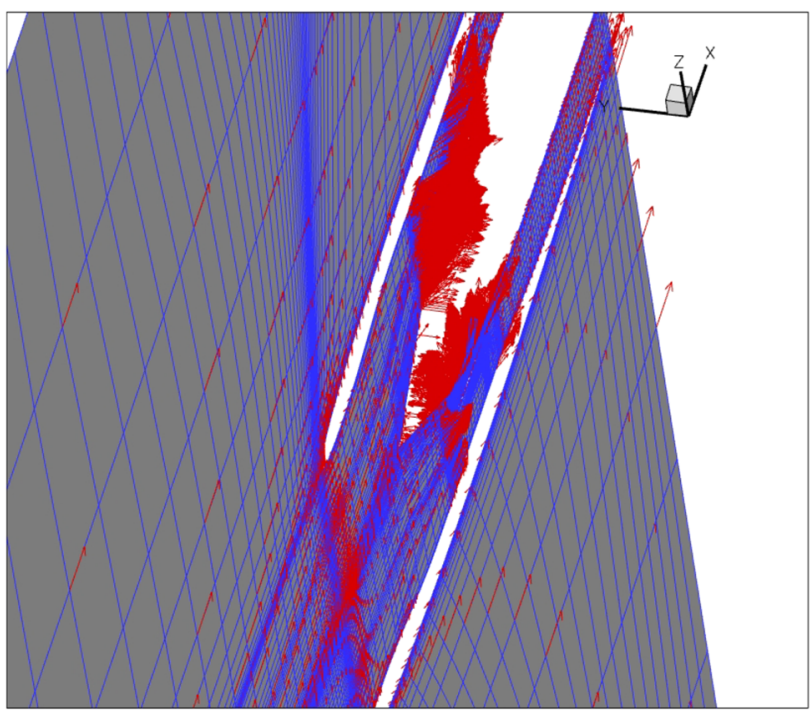

(b)

Fig. 5 Centerline velocity vectors for the $\mathrm{E}^{3}$ low pressure compressor. a Side view. b Orthogonal View

The grid structure used for the flow and particle analysis is shown in Fig. 4. The grid contained 12 structured, abutted grid blocks with a total of 327,583 nodes. The steady, viscous flow solution was generated for a Mach 0.8 cruise condition at $11,998 \mathrm{~m}$ assuming a standard warm day. Flow vectors along the centerline of the axi-symmetric solution are shown in Fig. 5.

The LEWICE3D ice particle analysis required several cloud input conditions and modeling parameters. The ice particle analysis assumed a free stream relative humidity of $0 \%$ and a free stream particle concentration of $0.3 \mathrm{~g} / \mathrm{m}^{3}$. The ice particles were assumed to be completely frozen and at the ambient temperature of the surrounding air $(229.3 \mathrm{~K})$. A simple particle impact and bounce model was used for this analysis because no model exists for the bounce and breakup of ice crystals typical of the HIWC environment. The simple particle bounce model assumed no breakup and no deposition. A coefficient of restitution of 1.0 and a coefficient of dynamic friction of 0.0 were used for the impact particle reflection model which yielded a lossless impact. This model was employed for all particle impacts in the engine and hence there was no buildup of ice. The ice particles were transported from the free stream through the compressor and out the compressor exit boundaries. Impingement efficiencies are reported for various surfaces. These were the net values that impinged upon the surface and do not represent the amount of ice that accreted on the surface. The actual values of accretion for all surfaces were zero because of the impact model employed (no deposition). Due to the bouncing model employed (multiple reflection and no deposition) the net amount of impingement on the engine solid surfaces (i.e. walls, blades, spinners, splitter lips, etc.) will be higher than that entering the engine. The amount of mass exiting the engine through the exit boundaries will however equal the amount entering the engine. The values are merely reported to give the reader information as to the amount and location of ice particle impingement.

It is worthwhile to report the definitions and equations used for the particle analysis. These include collection efficiency or impingement efficiency, $\beta$, average collection efficiency, $\beta_{\text {avg }}$, impingement rate $(I R)$, and the scoop factor $(S F)$. Impingement efficiency is a non-dimensional measure of the mass flux for a surface and is dependent upon the amount of convergence or dispersion of particles in a flow and the orientation of the surface relative to the particle paths. An impingement efficiency of one means the surface particle flux rate is equal to the free stream particle flux rate. A value less than one means the surface particle flux rate is less than the free stream particle flux rate and a value greater than one means that the surface particle flux rate is greater than the free stream level. The average collection efficiency is defined as:

$\beta_{\text {avg }}=\frac{\sum_{j=1}^{j=N} \beta_{j} \times A_{j}}{A_{\text {wetted }}}$

where $\mathrm{N}$ is the number of surface elements with nonzero impingement and $\beta_{\mathrm{j}}, A_{j}$ are the collection efficiency and area of the $j t h$ surface element respectively. The wetted area of the element, $A_{\text {wetted }}$, is the sum of the area of the elements which have non-zero impingement for which we have the equation:

$A_{\text {wetted }}=\sum_{j=1}^{j=N} A_{j}$

The impingement rate for a surface is defined as:

$I R=\beta_{\text {avg }} \times L W C_{\infty} \times V_{\infty} \times A_{\text {wetted }}$ 


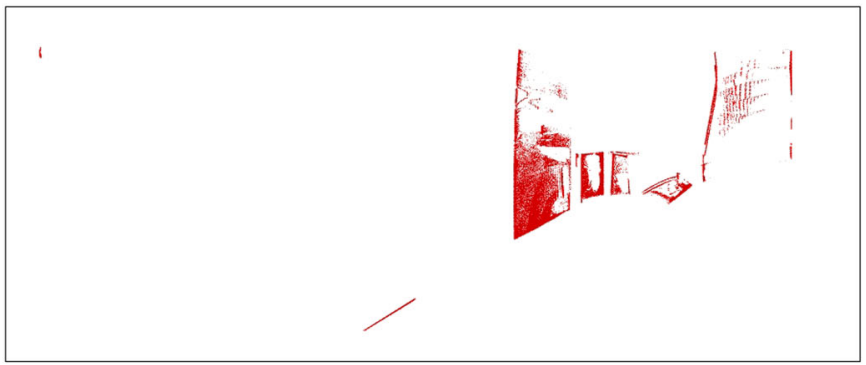

(a)

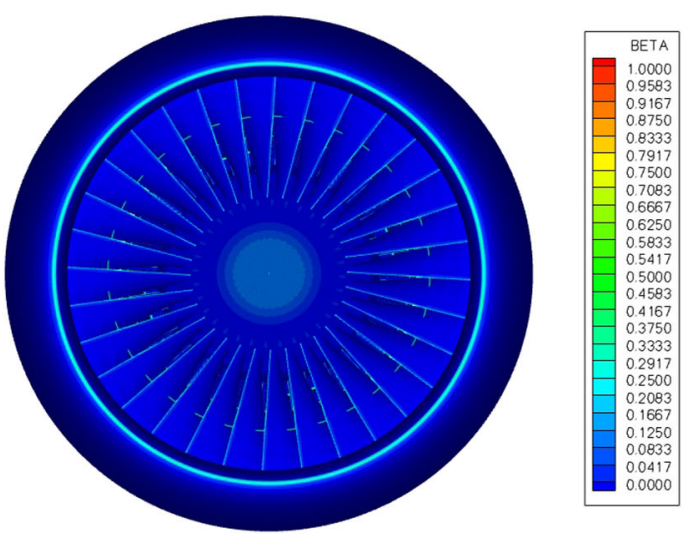

(b)
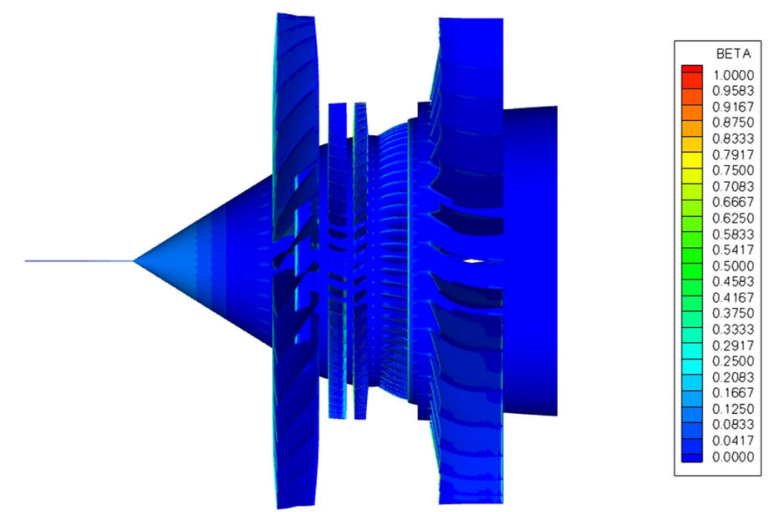

(c)
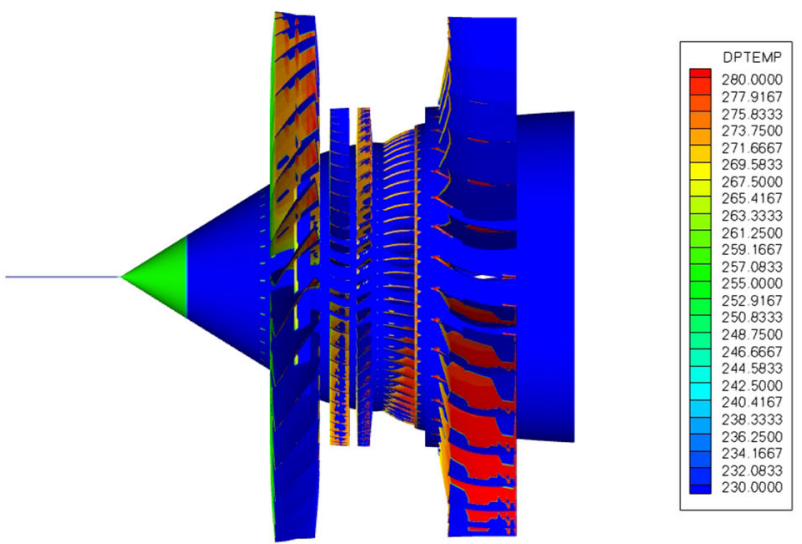

(d)

Fig. $6 \mathrm{E}^{3}$ particle transport results for a $5 \mu \mathrm{m}$ ice particle. a Particle parcel impact locations (axial view). b Impingement efficiency. c Impingement efficiency (axial view). d Particle impact temperature

where $L W C_{\infty}$ is the free stream liquid water content $(L W C)$ and $V_{\infty}$ is the free stream speed. The scoop factor, $S F$, is defined as the ratio of the mass impinging on a component divided by the mass available in the free stream for an area equal to the area bounded by the highlight of the inlet lip. The scoop factor is then:

$S F=\frac{I R}{I R_{\infty}}$

The free stream impingement rate $\left(I R_{\infty}\right)$ is defined as the rate at which the particles pass through an area traced out by the highlight of the inlet lip, $A_{\infty}$, traveling at the free stream speed, $V_{\infty}$, with an average collection efficiency of 1 and an $\mathrm{LWC}$ matching that of the free stream $\left(\mathrm{LWC}_{\infty}\right)$. The average collection efficiency for a surface is then:

$\beta_{\text {avg }}=\frac{I R \times A_{\infty}}{I R_{\infty} \times A_{\text {wetted }}}=S F \times \frac{A_{\infty}}{A_{\text {wetted }}}$

Figure 6 shows the particle parcel impact locations, impingement efficiency, and particle impact temperatures for the 5 micron particle case. From the ice particle trajectory impact points and impingement efficiency shown in Fig. 6a- c we can see that impingement occurs throughout the low pressure compressor. The ice particle parcel trajectory impact point plot (Fig. 6a) displays the impact locations for the particle parcels calculated in each of the zones for the mixing plane method. This plot illustrates the density and location of the impacting particle parcels used in the mixing plane method (each impact parcel is represented by a red triangle). The engine schematic shown in Fig. $4 \mathrm{c}$ is useful in clarifying the various elements which receive impingement. The peak value of average impingement efficiency for the various surface elements listed in Table 1 was for the inlet lip (0.30). The scoop factors were relatively small for the 5 micron particle for the various surface components shown in Table 1 $(<6 \%)$. The amount of mass entering the inner core was $16.260 \mathrm{~g} / \mathrm{m}^{3}$ which yielded a scoop factor of 0.0685 . Figure $3 \mathrm{~d}$ shows the temperature distribution of the impacting particles. As can be seen from the plot the impact temperatures of the particles increase as they pass through the compressor. The lowest impact temperatures from Fig. 6d are observed on the inlet lip while the highest were observed on splitter lip \#2 and the aft support strut. As the ice particles 
Table $1 \mathrm{E}^{3}$ transport statistics for an ice particle size of 5 microns

\begin{tabular}{lccccccc}
\hline Element & $\beta_{\text {avg }}$ & $\begin{array}{l}\text { Impingement } \\
\text { rate }(\mathrm{g} / \mathrm{s})\end{array}$ & Scoop factor & $\mathrm{D}_{\text {avg }}(\mu \mathrm{m})$ & $\mathrm{T}_{\text {avg }}(\mathrm{K})$ & Melt fraction $_{\text {avg }}$ & \% Evaporation \\
\hline Free stream capture tube* & 1.0000 & 237.397 & 1.0000 & 5.00 & 229.30 & 0.00 & 0.0 \\
Inlet lip & 0.3011 & 2.813 & 0.0118 & 4.93 & 256.20 & 0.00 & 4.9 \\
Inlet capture & 0.8618 & 183.492 & 0.7729 & 4.94 & 239.78 & 0.00 & 3.6 \\
Spinner & 0.0940 & 1.013 & 0.0043 & 4.89 & 256.23 & 0.00 & 6.6 \\
Fan blade & 0.0568 & 13.176 & 0.0555 & 4.85 & 263.32 & 0.02 & 8.8 \\
Splitter lip \#1 & 0.2151 & 1.189 & 0.0050 & 4.84 & 267.39 & 0.02 & 9.5 \\
IGV \#1 & 0.1024 & 3.630 & 0.0153 & 4.70 & 269.94 & 0.09 & 16.6 \\
Rotor \#1 & 0.0744 & 1.690 & 0.0071 & 4.80 & 271.12 & 0.12 & 11.0 \\
Splitter lip \#2 & 0.1582 & 0.793 & 0.0033 & 4.62 & 273.33 & 0.18 & 20.2 \\
Inner core & 0.7276 & 16.260 & 0.0685 & 4.59 & 272.26 & 0.06 & 22.6 \\
\hline
\end{tabular}

transport through the warming environment of the engine they increase in temperature and in some cases sublimate or melt and evaporate. The maximum average particle temperature for the 5 micron ice particle was $273.33 \mathrm{~K}$ (slightly above the freezing temperature of water) and occurred on splitter lip \#2 (Table 1). From the table we can also see that there was appreciable melting for splitter lip \#2 because the average melting fraction was greater than zero (0.18). The melting fraction is defined as the percentage of water mass to total mass. A melting fraction of one means the particle is entirely liquid. A melting fraction of zero means that the particle is fully frozen. We can also see from Table 1 that there was an appreciable amount of particle sublimation and evaporation for the 5 micron ice particles entering the engine core $(22.6 \%)$.

The particle impact, impingement efficiency, and temperature results for the 20 micron particle case are show in Fig. 7. As for the 5 micron ice particle the 20 micron ice particle revealed impingement throughout the low pressure compressor although the impingement area was larger for the 20 micron particle (Figs. 6c, 7c). For most of the components the impingement rates were higher for the 20 micron particle than for the 5 micron particle due to particle inertia (Tables 1, 2). Larger particles, which have larger inertia, are more resistive to changes in direction due to flow gradients than smaller particles which results in the larger particles being less apt to avoid obstacles. The amount of mass entering the inner core was $11.372 \mathrm{~g} / \mathrm{m}^{3}$ which yielded a scoop factor of 0.0479 . The mass entering the core for the 20 micron particle was smaller than for the 5 micron particle because the larger particles were less able to negotiate the turn into the inner core due to their larger inertia. The peak value of average impingement efficiency for the various surface elements listed in Table 2 was for splitter lip \#2 (0.3735). The scoop factors were larger for the 20 micron particle than for the 5 micron particle due to particle inertia. The maximum value of scoop factor for the 20 micron particle was for the fan blade (0.3748). From the temperature distributions in Fig. 7d and Table 2 we see that the maximum average temperature for the 20 micron particle was $270.70 \mathrm{~K}$ which occurred on splitter lip \#2. This was less than that for the 5 micron particle and is due to the thermal mass of the 20 micron particle being larger and hence more resistive to temperature change. We also see from Table 2 that there was no appreciable melting of the 20 micron particle and that there was a small amount of sublimation and evaporation for some of the elements $(<2.5 \%)$.

The particle impact, impingement efficiency, and temperature results for the 100 micron particle case are show in Fig. 8. For most of the components the impingement rates were higher for the 100 micron particle than for the 20 and 5 microns particles due to particle inertia (Tables 1, 2, 3). The amount of mass entering the inner core was $0.273 \mathrm{~g} / \mathrm{m}^{3}$ which yielded a scoop factor of 0.0012 . The mass entering the core for the 100 micron particle was smaller than for both the 5 and 20 microns particles because the larger particles were less able to negotiate the turn into the inner core due to their larger inertia. The peak value of average impingement efficiency for the various surface elements listed in Table 3 was for IGV \#1 (2.1457). The scoop factors were larger for the 100 micron particle than for the 20 and 5 microns particles due to particle inertia. The maximum value of scoop factor for the 100 micron particle was for the fan blade (0.8210). From the temperature distributions in Fig. 8d and Table 3 we see that the maximum average temperature for the 100 micron particle was $253.65 \mathrm{~K}$ which occurred at the exit of the inner core. This was less than that for the 20 and 5 microns particles and is due to the larger thermal mass of the 100 micron particles. We also see from Table 3 that there was no appreciable melting and mass loss due to sublimation and evaporation of the 100 micron particle for any of the elements. 


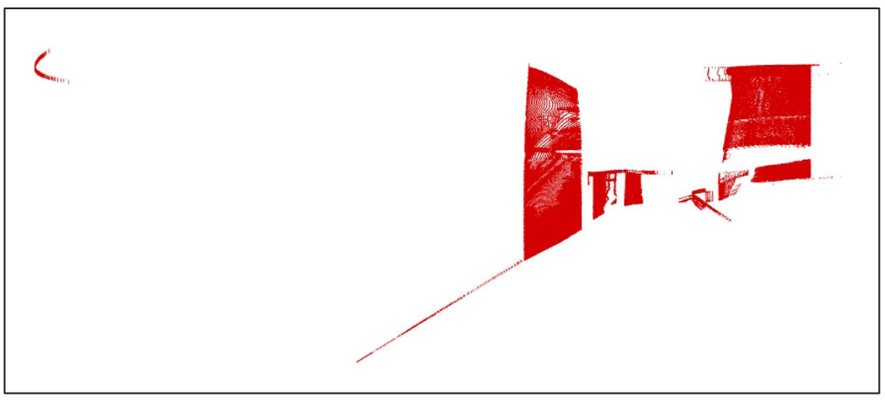

(a)

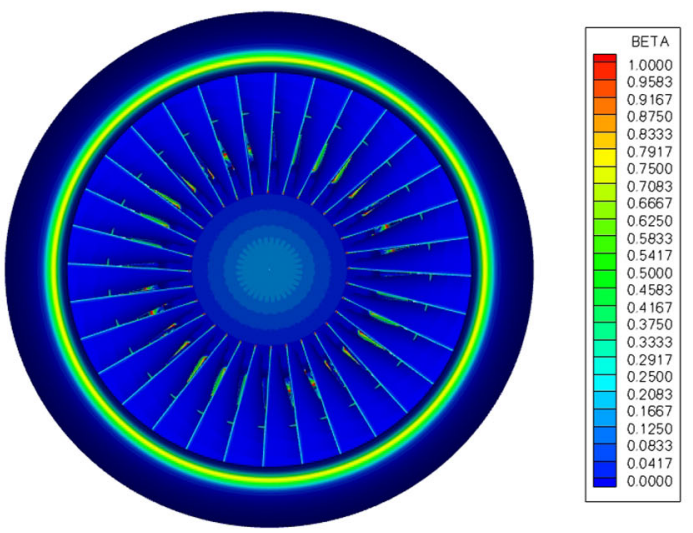

(b)
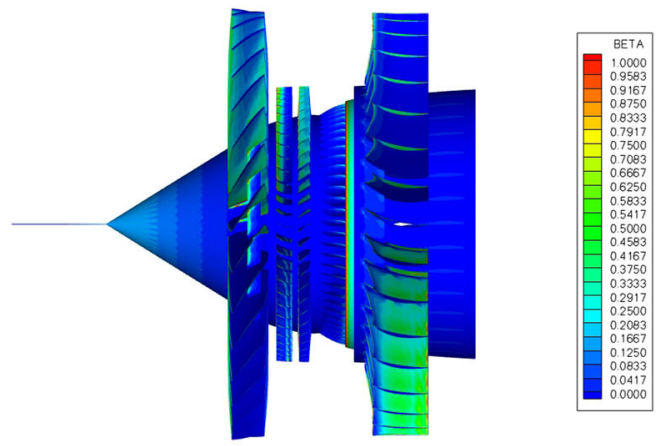

(c)
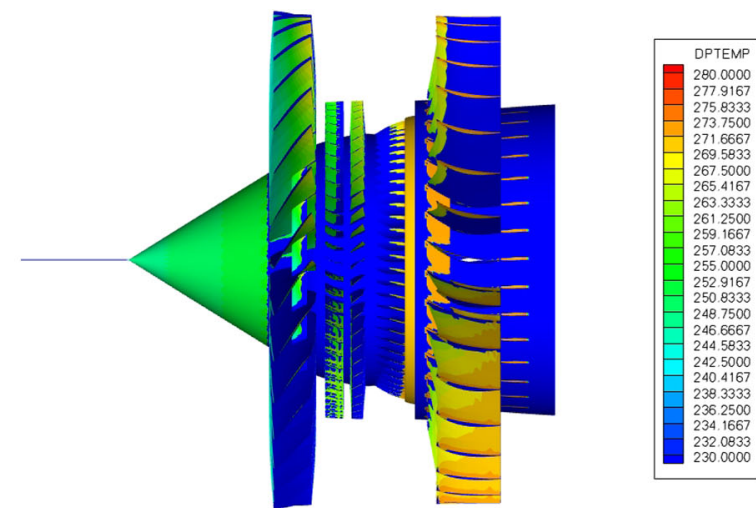

(d)

Fig. $7 \mathrm{E}^{3}$ particle transport results for a $20 \mu \mathrm{m}$ ice particle. a Particle parcel impact locations (axial view). b Impingement efficiency. c Impingement efficiency (axial view). d Particle impact temperature

Table $2 \mathrm{E}^{3}$ transport statistics for an ice particle size of 20 microns

\begin{tabular}{lccccccc}
\hline Element & $\beta_{\text {avg }}$ & $\begin{array}{l}\text { Impingement } \\
\text { rate }(\mathrm{g} / \mathrm{s})\end{array}$ & Scoop factor & $\mathrm{D}_{\text {avg }}(\mu \mathrm{m})$ & $\mathrm{T}_{\text {avg }}(\mathrm{K})$ & Melt fraction $\mathrm{avg}$ & \% Evaporation \\
\hline Free stream capture tube* & 1.0000 & 237.397 & 1.0000 & 20.00 & 229.30 & 0.00 & 0.0 \\
Inlet lip & 0.3097 & 24.306 & 0.1024 & 19.99 & 245.43 & 0.00 & 0.2 \\
Inlet capture & 0.9880 & 200.793 & 0.8458 & 19.98 & 239.03 & 0.00 & 0.3 \\
Spinner & 0.0787 & 4.592 & 0.0193 & 19.97 & 250.34 & 0.00 & 0.6 \\
Fan blade & 0.2552 & 88.968 & 0.3748 & 19.97 & 250.59 & 0.00 & 0.6 \\
Splitter lip \#1 & 0.1575 & 5.336 & 0.0225 & 19.97 & 257.53 & 0.00 & 0.7 \\
IGV \#1 & 0.2354 & 13.983 & 0.0589 & 19.97 & 253.40 & 0.00 & 0.6 \\
Rotor \#1 & 0.2750 & 8.888 & 0.0374 & 19.96 & 260.62 & 0.00 & 0.9 \\
Splitter lip \#2 & 0.3735 & 16.668 & 0.0702 & 19.91 & 270.70 & 0.00 & 0.00 \\
Inner core & 1.2586 & 11.372 & 0.0479 & 19.86 & 270.55 & 0.7 \\
\hline
\end{tabular}

\subsection{Computational performance}

The performance and elements of accuracy of the mixing plane analysis of the $\mathrm{E}^{3}$ engine will be discussed.
The $\mathrm{E}^{3}$ analysis was performed on a multi-processor Linux workstation. The mixing plane methodology which has been parallelized in LEWICE3D V3.5 using OpenMP [17] constructs has been shown to scale well to 20 processors. The 


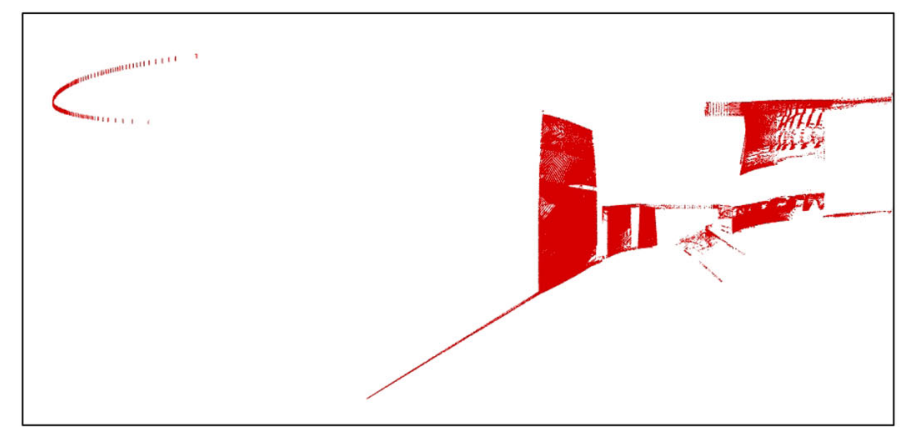

(a)
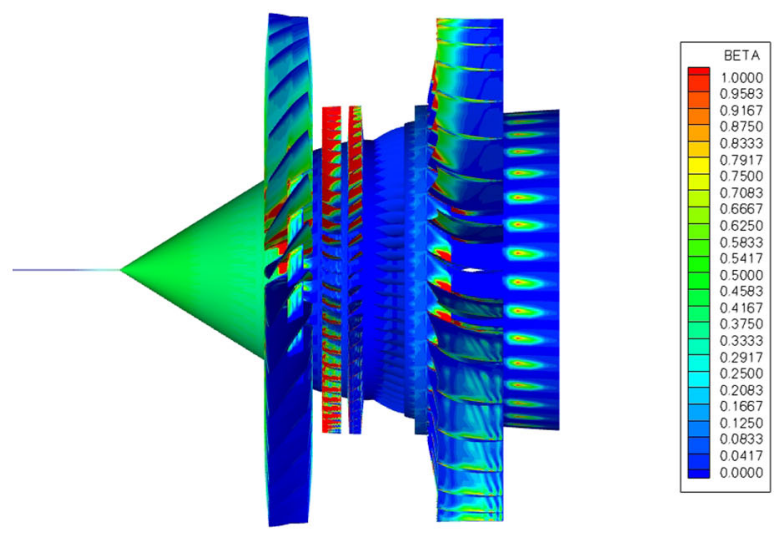

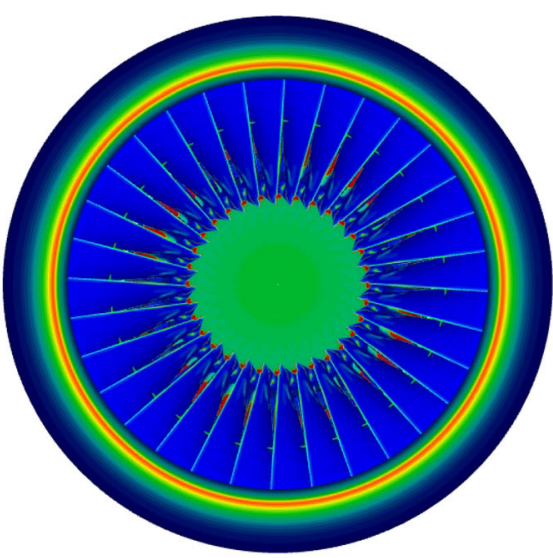

(b)

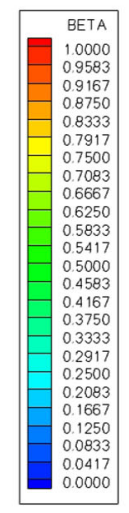

0.0000

(c)

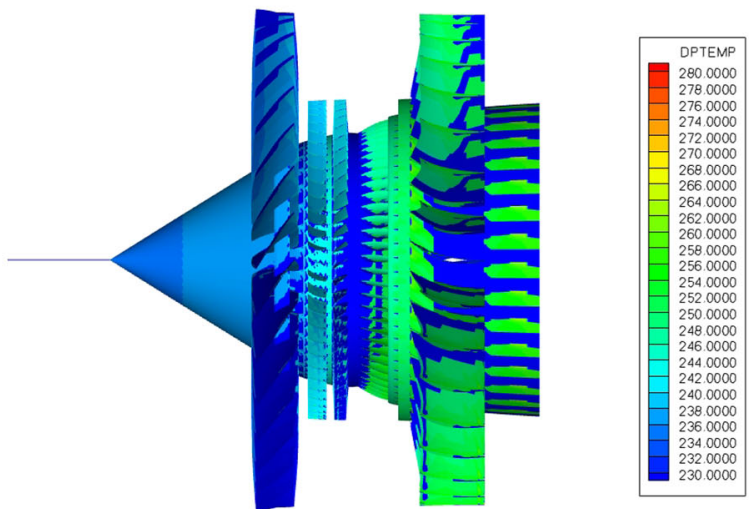

(d)

Fig. $8 \mathrm{E}^{3}$ particle transport results for a $100 \mu$ m ice particle. a Particle parcel impact locations (axial view). b Impingement efficiency. c Impingement efficiency (axial view). d Particle impact temperature

Table $3 \mathrm{E}^{3}$ transport statistics for an ice particle size of 100 microns

\begin{tabular}{lccccccc}
\hline Element & $\beta_{\text {avg }}$ & $\begin{array}{l}\text { Impingement } \\
\text { rate }(\mathrm{g} / \mathrm{s})\end{array}$ & Scoop factor & $\mathrm{D}_{\text {avg }}(\mu \mathrm{m})$ & $\mathrm{T}_{\text {avg }}(\mathrm{K})$ & Melt fraction avg & \% Evaporation \\
\hline Free stream capture tube* & 1.0000 & 237.397 & 1.0000 & 100.00 & 229.30 & 0.00 & 0.0 \\
Inlet lip & 0.2154 & 87.620 & 0.3691 & 100.00 & 232.21 & 0.00 & 0.0 \\
Inlet capture & 1.0891 & 228.496 & 0.9625 & 100.00 & 233.32 & 0.00 & 0.0 \\
Spinner & 0.5886 & 40.501 & 0.1706 & 100.00 & 237.02 & 0.00 & 0.0 \\
Fan blade & 0.3375 & 194.907 & 0.8210 & 100.00 & 237.49 & 0.00 & 0.0 \\
Splitter lip \#1 & 2.1457 & 72.070 & 0.3036 & 100.01 & 240.11 & 0.00 & 0.0 \\
IGV \#1 & 0.8313 & 92.055 & 0.3878 & 100.01 & 239.88 & 0.00 & 0.0 \\
Rotor \#1 & 0.6214 & 43.321 & 0.1825 & 100.01 & 241.98 & 0.00 & 0.0 \\
Splitter lip \#2 & 0.0131 & 0.626 & 0.0026 & 100.02 & 248.71 & 0.00 & 0.0 \\
Inner core & 0.0110 & 0.273 & 0.0012 & 100.03 & 253.65 & 0.00 & 0.1 \\
\hline
\end{tabular}

* Based on capture area of $3.26 \mathrm{~m}^{2}$

maximum execution time on an 8 processor machine was 1.7 hours for the 5 micron particle size which required 1,282,865 particle parcel trajectories. The memory required for the computations on an 8 processor machine was approximately $0.5 \mathrm{~GB}$.
Two issues that affect the accuracy of the method are the mass loss due to particle parcels exiting the volume grid and interpolation errors at the mixing planes. Parcels can exit the volume grid as a result of a rebound through the inflow boundary or in a corner. In both cases the mass is thrown 
away and tracked as total mass loss. The mass loss is typically greater for larger, more energetic particles which are less apt to be re-entrained in the flow if they are ejected from the surface towards an inflow boundary. The 100 micron case generated the maximum mass loss observed in the analysis of $1.94 \%$. The mass loss for both the 5 and 20 microns cases was $0.07 \%$. Interpolation errors at the mixing plane can result in a difference between the mass entering a mixing plane and the mass leaving a mixing plane. This error is larger for cases with larger radial gradients in mass. The maximum mass error was $2 \%$ for the 20 micron particle case at mixing plane 3 (the mixing plane between IGV \#1 and Rotor \#1).

Another general accuracy issue relates to the loss of information at the mixing plane due to the use of the steady flow assumption and circumferential averaging at the blade row exit. There was no way to evaluate the effect of this approximation in the current study although it can be inferred that the loss of information is greater when there are larger gradients in the particle properties at the exit plane. It also worth noting that the mixing plane approach to generating initial conditions for the particle parcels in a blade row is superior to lower fidelity methods which neglect the upstream effects and initialize the particle parcels with the local flow conditions and approximated concentrations at the inflow boundary [4].

\section{Conclusion}

Predictions for ice particle impingement efficiency, temperature, and melting fraction were generated for the $\mathrm{E}^{3}$ low pressure compressor using the Glenn Ice Particle Phase Change Model newly incorporated into LEWICE3D V3.5 and a flow solution from the ADPAC flow solver. The impingement efficiency results showed that as particle size increased average impingement efficiencies and scoop factors increased for the various components. The particle analysis also showed that the amount of mass entering the core decreased with increased particle size because the larger particles were less able to negotiate the turn into the inner core due to particle inertia. The particle phase change analysis results showed that the larger particles warmed less as they were transported through the low pressure compressor. Only the smallest 5 micron particles were warmed enough to produce melting with a maximum average melting fraction of 0.18 . The results also showed an appreciable amount of particle sublimation and evaporation for the 5 micron particles entering the engine core $(22.6 \%)$. These results suggest that the newly developed NASA Glenn mixing plane icing analysis method can be a useful tool for the analysis of turbo-machinery subject to water based mixed phase environments.
Acknowledgments The author would like to thank Joseph Veres, Philip Jorgenson and Russell Claus of the NASA Glenn Research Center, Cleveland, Ohio for contributing the flow solution for the $\mathrm{E}^{3}$ low speed compressor and for aid in interpreting turbo-machinery flow and particle transport physics. The NASA Aviation Safety Program, Atmospheric Environment Safety Technologies Project supported this work.

\section{References}

1. Mazzawy RS, Strapp JW (2007) Appendix D—an Interim Icing Envelope; SAE 2007-01-3311; SAE 2007 Aircraft and Engine Icing International Conference. Seville, Spain, November 2007

2. Mason J, Strapp W, Chow P (2006) The ice particle threat to engines in flight. In: 44th AIAA Aerospace Sciences Meeting, vol 4, pp 2445-2465

3. Federal Aviation Regulation (1974) Part 25, Airworthiness standard: transport category Airplanes, Appendix C. DOT, FAA, rev. 1982

4. Hamed A, Das K, Basu D (2005) Numerical simulations of ice droplet trajectories and collection efficiency on aero-engine rotating machinery. AIAA 2005-1248

5. Wright W, Jorgenson P, Veres J (2010) Mixed phase modeling in GlennICE with application to engine icing. AIAA 2010-7674

6. Steinthorsson E, Ameri AA, Rigby DL (1997) TRAF3D. MM-A multi-block flow solver for turbomachinery flows. AIAA-97-0996

7. Adamczyk JJ, Celestina ML, Beach TA (1989) Simulation of threedimensional viscous flow within a multistage turbine. ASME Paper 89-GT-152

8. Norment HG (1985) Calculation of water drop trajectories to and about three-dimensional lifting and non-lifting bodies in potential airflow. NASA CR-3935

9. Bidwell C (2012) Ice particle transport analysis with phase change for the E3 turbofan engine using LEWICE3D Version 3.2. AIAA 2012-3037

10. Krogh FT (1971) Variable order integrators for numerical solutions of ordinary differential equations. Report NPO-11643

11. Wright WB, Potapczuk MG, Levinson LH (2008) Comparison of LEWICE and GlennIce in the SLD Regime. AIAA 2008-0439

12. Bidwell CS, Potapczuk MG (1993) Users manual for the NASA Lewis three-dimensional ice accretion code (LEWICE3D). NASA TM-105974, December 1993

13. Hall E, Delaney R, Lynn S, Veres J (1988) Energy efficient low pressure subsystem aerodynamic analysis. NASA TM 1988-208402

14. Cline SJ, Halter PH, Kutney JT, Sullivan TJ (1983) Energy efficient engine: fan and quarter-stage component performance report. NASA CR 168070, Jan 1983

15. Steinbrenner JP, Chawner JR, Fouts CL (1990) The Gridgen 3D multiple block grid generation system. Final Report WRDC-TR90-3022, June 1990

16. Hall E, Heidegger N, Delaney R, ADPAC v1.0-User's manual. NASA CR 1999-206600

17. Open Architecture Review Board (2013) OpenMP application program interface, Version 4.0 - July 2013. OpenMP.org, July 2013, www.openmp.org 